\title{
Hemangiomatose capilar pulmonar, uma rara causa de hipertensão pulmonar. Primeiro caso brasileiro*
}

\author{
Pulmonary capillary hemangiomatosis. A rare cause of pulmonary hypertension. \\ The first Brazilian case
}

\author{
HELANO NEIVA DE CASTRO', MARA RÚBIA FERNANDES DE FIGUEIREDO', NORMA SELMA SANTOS², \\ TERESA NEUMA ALBUQUERQUE GOMES NOGUEIRA ${ }^{3}$, MARIA DA PENHA UCHOA ${ }^{4}$, \\ MARCELO ALCÂNTARA HOLANDA ${ }^{5}$
}

\begin{abstract}
RESUMO
A hemangiomatose capilar pulmonar é uma doença rara, caracterizada por proliferação de capilares que invadem o interstício pulmonar e o septo alveolar. Documentamos o primeiro caso brasileiro: um paciente do sexo masculino, de 21 anos, com hipertensão pulmonar grave, que evoluiu para óbito. Na tomografia computadorizada de alta resolução apresentava pequenas opacidades intersticiais nodulares maldefinidas, bilateralmente. Foi realizada biópsia pulmonar post-mortem e encontrada intensa proliferação multifocal de capilares nas paredes alveolares, septos interlobulares e tecido conjuntivo peribrônquico. 0 diagnóstico de hemangiomatose capilar pulmonar deve ser considerado nos pacientes com hipertensão pulmonar e alterações sugestivas na tomografia computadorizada de alta resolução.
\end{abstract}

Descritores: Hemangioma capilar/etiologia; Hemangioma capilar/diagnóstico; Hipertensão pulmonar/complicações; Tomografia computadorizada de emissão; Relato de caso

\begin{abstract}
Pulmonary capillary hemangiomatosis is a rare disorder characterized by a proliferation of capillaries that invade the pulmonary interstitium and alveolar septae. Herein, we report the first Brazilian case of pulmonary capillary hemangiomatosis. A 21-year-old man presented with severe pulmonary hypertension that eventually resulted in his death. Upon admission, a computed tomography scan of the chest revealed diffuse ill-defined bilateral pulmonary nodules. A postmortem lung biopsy revealed pronounced multifocal proliferation of capillaries in the alveolar walls, interlobular septa and peribronchial connective tissue. A diagnosis of pulmonary capillary hemangiomatosis should be considered in patients presenting pulmonary hypertension and suspicious changes on high-resolution computed tomography scans.
\end{abstract}

Keywords: Hemangioma, capillary/etiology; Hemangioma capillary/diagnosis; Hypertension, pulmonary/ complications; Tomography, Emission-Computed; Case report

\footnotetext{
* Trabalho realizado no Serviço de Pneumologia do Hospital de Messejana - HM - Fortaleza (CE) Brasil.

1. Médico Residente de Pneumologia do Hospital de Messejana - HM - Fortaleza (CE) Brasil.

2. Médica Radiologista do Hospital de Messejana - HM - Fortaleza (CE) Brasil.

3. Médica Patologista da Faculdade de Medicina da Universidade Federal do Ceará - UFC - Fortaleza (CE) Brasil.

4. Médica Pneumologista do Hospital de Messejana - HM - Fortaleza (CE) Brasil.

5. Chefe da Unidade de Terapia Intensiva Respiratória do Hospital de Messejana - HM - Fortaleza (CE) Brasil.

Endereço para correspondência: Helano Neiva de Castro. Rua Gilberto Studart 488, 1.265, apto 201. Parque do Cocó, Fortaleza - CE. CEP: 60190-750. Tel: 5585 3265-6619. E-mail: helanocastro@terra.com.br

Recebido para publicação em 17/2/05. Aprovado, após revisão, em 15/4/05.
} 


\section{INTRODUÇÃO}

A hemangiomatose capilar pulmonar (HCP) é uma doença rara, que se caracteriza basicamente por uma proliferação exagerada de capilares no interstício pulmonar e septos alveolares. ${ }^{(1)}$ Ocorre em ambos os sexos, na faixa etária dos doze aos 71 anos e apenas um total de 31 casos foram descritos na literatura internacional ${ }^{(2)}$. Este relato tem como objetivo apresentar o primeiro caso brasileiro de hipertensão pulmonar grave secundária a hemangiomatose capilar pulmonar, bem como uma revisão dos aspectos patogênicos, diagnósticos e terapêuticos envolvidos.

\section{RELATO DO CASO}

Um paciente do sexo masculino, com 21 anos de idade, motorista, residente em área rural (Viçosa - CE) procurou serviço médico queixando-se de dispnéia progressiva aos esforços havia um ano, com episódios isolados de hemoptise.

Quinze dias antes da admissão hospitalar fez um cateterismo cardíaco que evidenciou hipertensão pulmonar com pressão sistólica de artéria pulmonar de $56 \mathrm{mmHg}$. Ao exame físico inicial observava-se dispnéia $(+/ 4+)$ e segunda bulha hiperfonética em foco pulmonar. Negava tuberculose, asma, tabagismo e uso de drogas ilícitas.

Os exames de hemograma, função hepática e função renal foram normais. A gasometria arterial em ar ambiente apresentou: $\mathrm{pH}$ de 7,$44 ; \mathrm{PaCO}_{2}$ de 31; $\mathrm{PaO}_{2}$ de 67; $\mathrm{SaO}_{2}$ de $93 \%$ e $\mathrm{HCO}_{3}$ de 20 . Os exames parasitológico de fezes, fator antinúcleo, anticoagulante lúpico, anticardiolipina e antivírus da imunodeficiência humana foram negativos. A pesquisa seriada de bacilo álcool-acidorresistente no escarro induzido foi negativa.

0 eletrocardiograma mostrava sinais de sobrecarga ventricular direita. 0 exame de ecocardiograma evidenciava uma fração de ejeção de 79\%, dilatação das câmaras direitas, insuficiência tricúspide moderada e pressão média de artéria pulmonar de $56 \mathrm{mmHg}$. Pelo agravamento dos sintomas fez-se uma nova arteriografia pulmonar, a qual mostrou pressão sistólica de artéria pulmonar de $80 \mathrm{mmHg}$, pressão diastólica de artéria pulmonar de $42 \mathrm{mmHg}$, pressão média de artéria pulmonar de $55 \mathrm{mmHg}$ e pressões de câmaras cardíacas direitas elevadas.

Foram realizadas espirometria, ultra-sonografia de abdome com doppler e biópsia retal com pesquisa de esquistossomose, cujos resultados apresentaram-se normais. A radiografia de tórax inicial foi considerada normal (Figura 1A). Contudo, na tomografia computadorizada de alta resolução foi notado um padrão de vidro fosco difuso com pequenas opacidades nodulares bilaterais, de limites pouco definidos (Figura 2).

0 paciente apresentou piora clínica com franca insuficiência respiratória e elevação da pressão arterial pulmonar (pressão sistólica de artéria pulmonar de $90 \mathrm{mmHg}$ ) e piora radiológica (Figura 1B). Foi transferido para unidade de terapia intensiva e submetido a pulsoterapia com metilprednisolona ( $1 \mathrm{~g} / 3$ dias), sem resposta clínica efi-

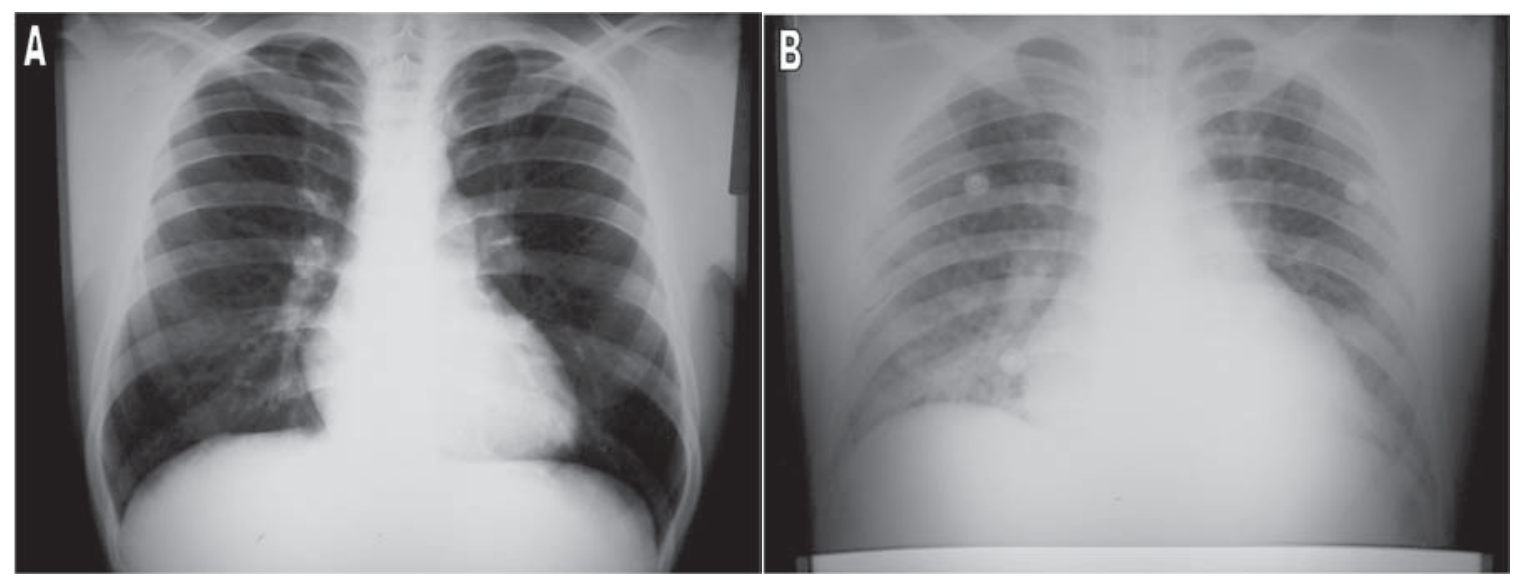

Figura 1 - A) Radiografia de tórax inicial aparentemente normal; B) Radiografia de tórax na admissão da UTI, com sinais de congestão e hipertensão pulmonar." 

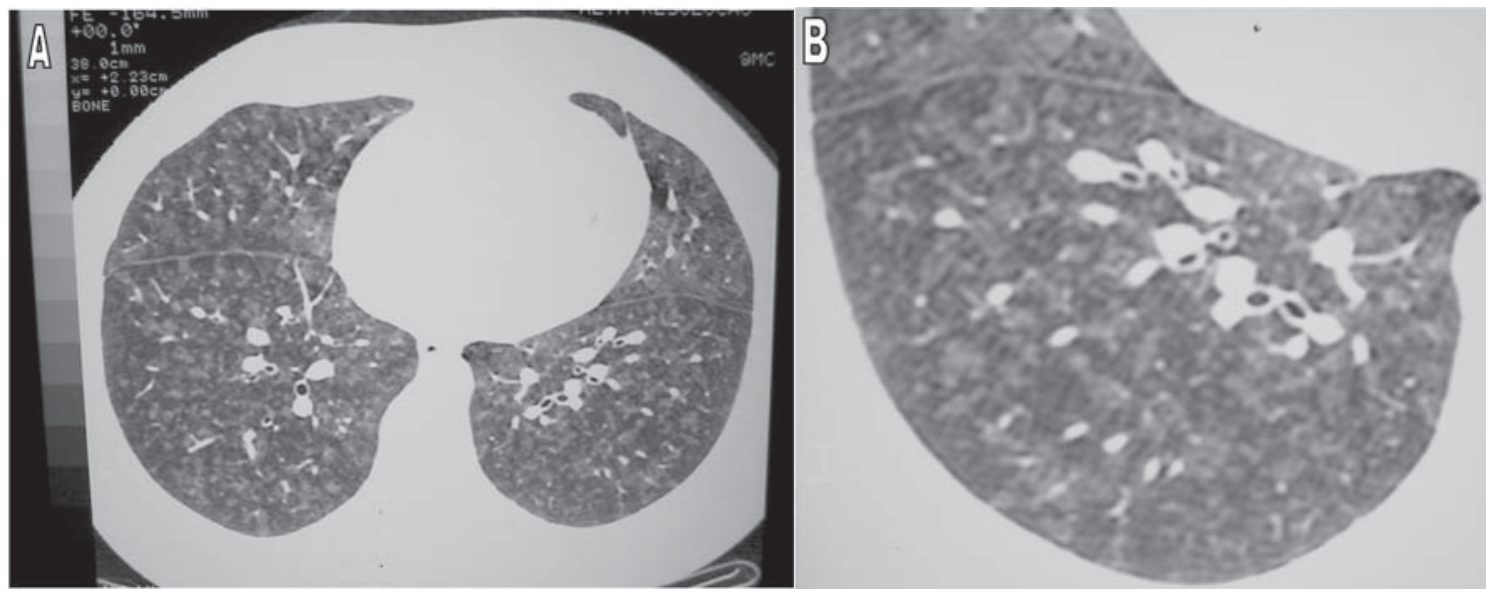

Figura 2 - A e B) TCAR mostrando opacidades nodulares bilaterais de limites imprecisos e vidro fosco difuso.

caz. Foi submetido à ventilação mecânica invasiva, mas não houve melhora na hipoxemia, tendo-se seguido o óbito.

Na biópsia pulmonar post-mortem, encontrouse intensa proliferação multifocal de capilares nas paredes alveolares, septos interlobulares, em região peribrônquica e perivascular, assim como na pleura. Hemossiderose e espessamento discreto da média das artérias pulmonares e da camada íntima em vênulas e pequenas veias foram observados (Figura 3). Notaram-se também trechos com fibrose septal discreta e leucócitos esparsos. Este conjunto de achados é consistente com o diagnóstico de hemangiomatose capilar pulmonar. ${ }^{(3-9)}$

\section{DISCUSSÃO}

A HCP é uma rara causa de hipertensão pulmonar. Apenas 31 casos já foram relatados na literatura. Este é o $32^{\circ}$ caso documentado. Não há predominância por $\operatorname{sexo}^{(3-8)}$ e ocorre principalmente em adultos jovens (variando dos seis aos 71 anos de idade).

A sua etiologia é desconhecida, mas em alguns casos sugere-se relação com o uso de anticoncepcionais orais e em outros um componente familiar. ${ }^{(8-}$ 11). Há ainda evidências de que possa resultar de uma proliferação capilar neoplásica ou ser conseqüente a uma infecção prévia. ${ }^{(12-15)}$

Os pacientes apresentam-se clinicamente com dispnéia, hemoptise e nos casos mais avançados com hipertensão pulmonar. Achados espirométricos inespecíficos, tanto de restrição volumétrica quanto de obstrução ao fluxo aéreo, além de capacidade de difusão de monóxido de carbono diminuída, aparecem nas provas de função pulmonar ${ }^{(15)}$.

Os achados radiológicos mais típicos são de micronódulos, infiltrados reticulonodulares difusos e aumento do calibre da artéria pulmonar. Na tomografia computadorizada de tórax de alta resolução apresentam-se com padrão de vidro fosco, alargamento da artéria pulmonar e presença de pequenos nódulos difusos e bilaterais maldefinidos. ${ }^{(15-17)}$

No exame histopatológico verifica-se: proliferação multifocal de capilares nas paredes alveolares, septos interlobulares, peribrônquicos e pleurais; hipertrofia da musculatura das arteríolas pulmonares; espessamento da camada íntima de vênulas; e hemossiderose. ${ }^{(17-18)} 0$ diagnóstico diferencial deve ser feito com a doença veno-oclusiva pulmonar, que cursa com trombose e fibrose obliterando vênulas e com conseqüente congestão pulmonar. ${ }^{(19-20)}$ Em alguns casos de doença venooclusiva pulmonar podem ser encontrados focos de HCP. 0 inverso também pode ocorrer, ou seja, podem ser encontrados focos de doença venooclusiva pulmonar na HCP, mas não de modo disseminado. 1sto torna a distinção entre HCP e doença veno-oclusiva pulmonar em certos casos difícil e dependente da determinação da característica dominante. Uma congestão pulmonar pode levar a um ingurgitamento dos capilares alveolares e a uma falsa impressão de HCP; contudo, neste caso, não é observado aumento quantitativo dos capilares $^{(9)}$.

Tentativas de tratamento com esteróides e 


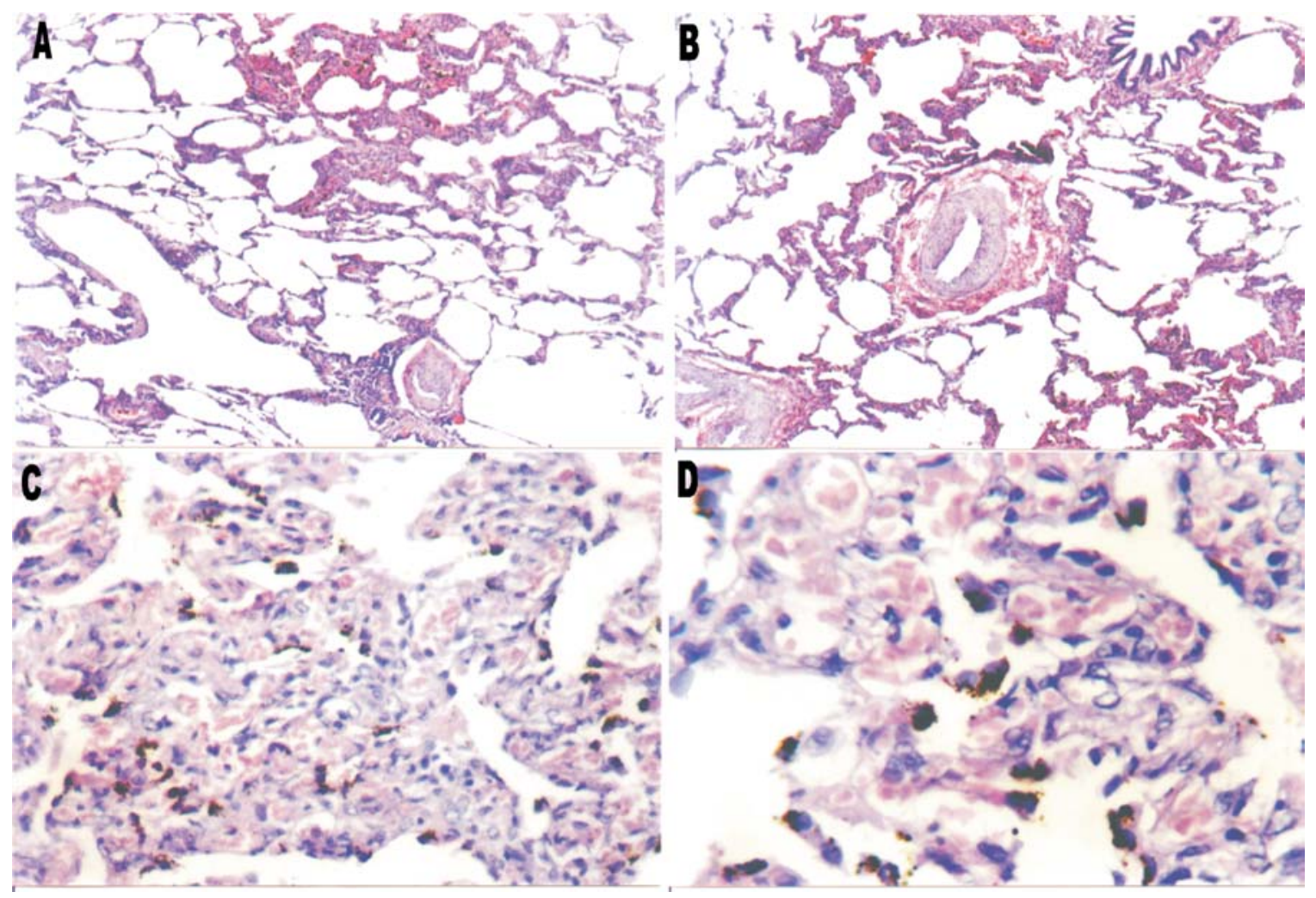

Figura 3 - Espessamento de septos alveolares e peribrônquicos, por proliferação de capilares que se encontram congestos (A e B, HE-100x), hemossiderose e proliferação de capilares nos septos (C e D, HE-400x).

ciclosporina não obtiveram sucesso. Terapias com alfa-interferon, ${ }^{(17)}$ pneumectomia na doença unilateral ${ }^{(10)}$ e transplante de pulmão também foram relatados ${ }^{(18)}$.

Neste caso, inicialmente pensamos em otimizar os níveis de pressão da artéria pulmonar com o uso de vasodilatadores, após um teste de vasodilatação com óxido nítrico, para uma posterior biópsia pulmonar a céu aberto. Contudo esta conduta foi abandonada pois o uso de vasodilatadores na suspeita de HCP pode levar a um risco de edema agudo de pulmão durante o procedimento. ${ }^{(13)}$

Em apenas dois casos documentados na literatura houve respostas terapêuticas satisfatórias. 0 primeiro com o uso de alfa-interferon, sem recorrência após cinco anos, e o segundo com o uso de doxiciclina. ${ }^{(2)}$ Neste último, o paciente apresentava uma manifestação de endoteliose atípica e inicialmente não respondeu ao tratamento com alfa-interferon. A resposta foi acompanhada pela dosagem urinária do fator de crescimento do fibroblasto, sendo esta a melhor resposta terapêutica dos casos já documentados na literatura.

0 prognóstico da HCP em geral é ruim, com sobrevida após o diagnóstico, sem tratamento, menor que dois anos. ${ }^{(1-18)}$

Devido à raridade desta patologia, observamos, após revisão, que este é o primeiro caso a ser publicado na literatura médica brasileira indexada. Cabe ressaltar a importância de se incluir a HCP no diagnóstico diferencial de hipertensão pulmonar, para que novos casos sejam precocemente diagnosticados.

\section{REFERÊNCIAS}

1. Case records of the Massachusetts General Hospital: weekly clinicopathological exercises; case 38-2000. A 45-year-old woman with exertional dyspnea, hemoptysis, and pulmonary nodules. N Engl J Med. 2000;343(24):1788-96.

2. Gins LC, Robert DH, Mark EJ, Brusch JL, Marler JJ. Pulmonary capillary hemangiomatosis with atypical endotheliosis: successful antiangiogenic therapy with doxycycline. Chest. 2003;124(5):2017-22. 
3. Fernandez-Alonso J, Zulueta T, Reyes-Ramirez JR, Castillo-Palma MJ, Sanchez-Roman J. Pulmonary capillary hemangiomatosis as cause of pulmonary hypertension in a young woman with systemic lupus erythematosus. J Rheumatol. 1999;26(1):231-3.

4. Eltorky MA, Headley AS, Winer-Muram H, Garret HE Jr, Griffin JP. Pulmonary capillary hemangiomatosis: a clinico-pathologic review. Ann Thorac Surg. 1994; 57(3):772-6.

5. Ishii H, lwabuchi K, Kameya T, Koshino H. Pulmonary capillary haemangiomatosis. Histopathology. 1996;29(3):275-8.

6. Masur Y, Remberger K, Hoefer M. Pulmonary capillary hemangiomatosis as a rare cause of pulmonary hypertension. Pathol Res Pract. 1996;192(3):290-5; discussion 296-9. Erratum in: Pathol Res Pract. 1996;192(6):646.

7. Wagenvoort CA, Beetstra A, Spijker J. Capillary hemangiomatosis of the lungs. Histopathology. 1978;2(6):401-6.

8. Blobe GC, Schiemann WP, Lodish HF. Role of transforming growth factor beta in human disease. $\mathrm{N}$ Engl J Med. 2000;342(18):1350-8.

9. Travis WD, Colby TV, Koss MN, Müller NL, King TE, Christenson ML. Non-neoplastic disorders of the lower respiratory tract. Atlas of nontumor pathology. Washington, DC: The American registry of pathology; 2002. p.788-9. Chapter 15. Travis WD. Pulmonary hypertension and other vascular disorders.

10. Domingo C, Encabo B, Roig J, Lopez D, Morera J. Pulmonary capillary hemangiomatosis: a case report and review of the literature. Respiration. 1992;59(3):178-80.

11. Dufour B, Maitre S, Humbert M, Capron F, Simonneau G, Musset D. Hight-resolution CT of the chest in four patents with pulmonary capillary hemangiomatosis or pulmonary venoocclusive disease. AJR Am J Roentgenol. 1998;171(5):1321-4

12. Heath D, Edwards JE. The pathology of hypertensive pulmonary vascular disease. Circulation 1958;(4)18:533-47.

13. Humbert M, Maitre S, Capron F, Rain B, Musset D, Simonneau G. Pulmonary edema complicating continuous intravenous prostacyclin in pulmonary capillary hemangiomatosis. Am J Respir Crit Care Med. 1998;157(5 Pt 1):1681-5.

14. Langleben D, Heneghan JM, Batten AP, Wang NS, Fitch $\mathrm{N}$, Schlesinger RD. Familial pulmonary capillary hemangiomatosis resulting in primary pulmonary hypertension. Ann Intern Med. 1988;109(5):439.

15. Magee F, Wright JL, Kay JM, Peretz D, Donevan R, Chung A. Pulmonary capillary hemangiomatosis. Am Rev Respir Dis. 1985;132(4):922-5.

16. Tron V, Magee F, Wright JL, Colby T, Churg A. Pulmonary capillary hemangiomatosis. Hum Pathol. 1986;17(11):114450.

17. White CW, Sondheimer HM, Crouch EC, Wilson H, Fan LL. Treatment of pulmonary hemangiomatosis with recombinant interferon alfa-2a. N Engl J Méd. 1989;320(18):1197-200.

18. Faber CN, Yousem AS, Dauber JH, Griffith BP, Hardesty RL, Paradis IL. Pulmonary capillary hemangiomatosis: a report of three cases and a review of the literature. Am Rev Respir Dis. 1989;140(3):808-13.

19. Holcomb BW Jr, Loyd JE, Ely EW, Johnson J, Robbins IM. Pulmonary veno-occlusive disease: a case series and new observations. Chest. 2000;118(6):1671-9.

20. Almagro P, Alvarez M, Garau J. Lung biopsy in pulmonary veno-occlusive disease. Chest. 2001;120(4):1421-2. 